\title{
Is missing Fe I opacity in stellar atmospheres a significant problem?
}

\author{
F. Castelli1 ${ }^{1,2}$ and R. L. Kurucz ${ }^{3}$ \\ 1 Istituto di Astrofisica Spaziale e Fisica Cosmica, INAF, via del Fosso del Cavaliere, 00133 Roma, Italy \\ 2 Osservatorio Astronomico di Trieste, via G.B. Tiepolo 11, 34131 Trieste, Italy \\ ${ }^{3}$ Harvard-Smithsonian Center for Astrophysics, 60 Garden Street, Cambridge, MA 02138, USA \\ e-mail: rkurucz@cfa.harvard.edu
}

Received 16 June 2003 / Accepted 16 February 2004

\begin{abstract}
We present an empirical model-atmosphere investigation of missing Fe I opacity. Houdashelt et al. (2000) estimated that if Dragon \& Mutschlecner (1980) Fe I cross sections used in the MARCS model atmospheres (Gustafsson et al. 1975) were replaced by the Bautista (1997) cross sections the solar continuous flux would be reduced by $15 \%$ in the near ultraviolet. That would imply systematic errors in models for F, G, and K stars. As a consequence, since ATLAS9 (Kurucz 1993a) uses an approximation to the same Dragon \& Mutschlecner (1980) opacities, there should also be similar systematic errors in ATLAS9 models that required this investigation. Bound-free Fe I cross sections computed by Bautista (1997) in the framework of the IRON Project were used to generate the continuous Fe I absorption coefficient. It was incorporated in the Kurucz (1993a) ATLAS9 code, in place of that currently used, which is based on approximate cross sections by Kurucz. By combining Opacity Distribution Functions (ODFs) computed without the contribution of Fe I autoionization lines with the new Fe I absorption coefficient which is crowded with autoionization resonances, we obtained solar metallicity model atmospheres and energy distributions for several combinations of $T_{\text {eff }}$ and $\log g$. The comparison of these models with the standard ATLAS9 models has shown that there are no differences in the $T$ - $\tau_{\text {Ross }}$ relations, while there are some changes in the energy distributions for $T_{\text {eff }} \leq 7000 \mathrm{~K}$, but limited to small wavelength regions around $2150 \AA$, where Kurucz has less opacity, and $3350 \AA$, where Bautista has less opacity. The differences are of the order of $25 \%$ and less than $10 \%$, respectively. That around $2150 \AA$ disappears for $T_{\text {eff }} \leq 5500 \mathrm{~K}$ owing to the fall of the emergent flux at these wavelengths in cool stars. This behaviour is independent of the gravity. The explanation is that our line list actually has more autoionizing opacity than Bautista's but it is treated as bound-bound line opacity rather than as bound-free opacity.
\end{abstract}

Key words. stars: atmospheres - atomic processes - atomic data

\section{Introduction}

Continuous opacities are one of the ingredients for the computation of stellar model atmospheres. Their accuracy depends on the availability of reliable cross sections for the bound-free transitions involving atomic or molecular levels. While experimental data are not very numerous, there are sets of computed cross sections for several atoms, in particular thanks to the efforts of the Opacity Project Team (OP, Seaton et al. 1994) and Iron Project Team (IP, Hummer et al. 1993)

Bautista \& Pradhan (1995) showed that the Fe I photoionization cross sections are underestimated by more than three orders of magnitude when coupling effects and resonances are ignored in the computations. Bautista (1997), in the framework of the Iron Project, performed large-scale close coupling calculations using the R-matrix method and obtained

Send offprint requests to: F. Castelli, e-mail: castelli@ts.astro.it
Fe I photoionization cross sections which incorporate numerous autoionization lines with complex structures.

Houdashelt et al. (2000) estimated that if the Fe I cross sections from Dragon \& Mutschlecner (1980), included in the MARCS model atmospheres (Gustafsson et al. 1975) were replaced by the Bautista (1997) cross sections, the solar continuous flux would be reduced by $15 \%$ in the near ultraviolet around $3100 \AA$. As a consequence, this would imply systematic errors in the current models for F, G, and $\mathrm{K}$ stars which could be removed once different Fe I photoionization cross section are used in the models. In addition, the problem of the "missing UV opacity" would be solved. Bell et al. (2001) investigated this possibility and computed the solar flux in the region 2000-4000 ̊ by adopting the Fe I continuous cross sections from Bautista (1997) and the line opacity measured in the observed solar spectrum. They found that the computed flux is still larger than the observed one and that the two fluxes become comparable only if the Fe I opacity from Bautista is doubled. 
We have extended in this paper the study of Bell et al. (2001) to models for F, G, and K stars and have investigated how large are the changes in the model structure and in the low resolution fluxes when Fe I continuous cross sections used in the ATLAS9 (Kurucz 1993a) model atmosphere code are replaced by Bautista's (1997) Fe I cross sections. We used in this study Kurucz's codes, but the results would be the same when other model atmospheres programs employing Fe I continuous opacity from Dragon \& Mutschlecner (1980) and line opacity from Kurucz (1994) are used.

\section{Fe I continuous absorption coefficient}

The Fe I continuous absorption coefficient was computed in the Kurucz codes with $48 \mathrm{Fe}$ I terms, which were taken from Corliss \& Sugar (1982). Dragon \& Mutschlecner (1980) computed the Fe I absorption coefficient by using the first 39 . Table 1, Col. 2, lists the Fe I configurations, Cols. 4-7 the data from the Kurucz codes, and Cols. 8-11 the data we used for the Fe I b-f absorption coefficient based on the Bautista (1997) cross sections. In particular, Cols. 4 and 8 show the term energies $E_{n}$, Cols. 5 and 9 the parent term according to Corliss \& Sugar (1982) and Bautista (1997), respectively, Cols. 6 and 10 the ionization energy $E_{\text {ion }}$ to the parent term, Cols. 7 and 11 the wavelength $\lambda_{\text {ion }}$ corresponding to $E_{\text {ion }}$. For computing the absorption coefficient from the Bautista (1997) cross sections we replaced the approximate values $E_{n}$ and $E_{\text {ion }}$ of Cols. 4 and 6 with weighted means based on the observed energies (Nave et al. 1994). The weights are the $g_{n}$ statistical weights, whose sum is given in Table 1, Col. 3. For terms having in Col. 1 Nos. 7, 17, and 38, cross sections from Bautista (1997) were not used and those from Kurucz were kept. The reason is a mis-match in the Bautista file for the ${ }^{3} \mathrm{H}$ terms and a too large discrepancy between the observed and calculated $\lambda_{\text {ion }}$ for term number 38 (last column).

Columns 5 and 9 in Table 1 show that for several Fe I configurations the parent terms are different in Kurucz and in Bautista. According to Bautista (1997), three Fe II parents (5D) $4 \mathrm{~s} \mathrm{a}^{6} \mathrm{D}, 3 \mathrm{~d}^{7} \mathrm{a}^{4} \mathrm{~F}$, and $3 \mathrm{~d}^{7} \mathrm{a}^{2} \mathrm{G}$ dominate for the configurations considered here. In Corliss \& Sugar (1982) and in Kurucz the parent listed is the parent of the strongest component of the term. In Bautista (1997) the parent is the parent of the lowest significant component of the term that contributes to the photoionization. We listed in Cols. 12 and 13 the calculated $E_{\text {ion }}$ to the parent term and the corresponding $\lambda_{\text {ion }}$ derived from Table 4 in Bautista (1997). The last column shows that the differences between the measured and calculated $\lambda_{\text {ion }}$ range from few angstroms up to more than $2000 \AA$, as in the case of the term $3 \mathrm{~d}^{7} 4 \mathrm{~s} \mathrm{~d} \mathrm{~d}^{3} \mathrm{~F}$ (No. 38 in Table 1). We adopted as ionization threshold the smaller of the measured and computed wavelengths.

\section{Fe I cross sections}

Fe I cross sections from Kurucz and from Bautista (1997), $\mathrm{Fe}$ I autoionization lines from Kurucz, and line cross sections from Bautista derived by subtracting an approximate background continuum are discussed and compared in this section. Figure 1 displays these cross sections for 6 out of the $48 \mathrm{Fe}$ I terms given in Table 1. They have numbers 1, 9, 18, 26, 34, and 42 (Col. 1, Table 1). For each term there are two panels, an upper plot and a lower plot, each showing 4 different cross sections. The upper plot displays the Bautista cross sections as taken from his files (thin blue line), the Bautista cross sections with estimated continuum subtracted, leaving autoionizing lines only (thick blue line), Kurucz autoionizing lines (thin pink line), Kurucz autoionizing lines smoothed (red line). The lower plot shows the estimated Bautista continuum (green line), the Fe I continuum from the Kurucz approximation (yellow line), the average line cross section from Bautista and from Kurucz for each "big" wavelength interval in the ODFs (blue histogram and red histogram, respectively). Results and conclusions on cross sections do not change significantly for terms in Table 1 different from those we have plotted.

\subsection{Fe I photoionization cross sections from Kurucz}

We first make clear the real behavior of the opacity that is approximated in the Kurucz codes. A photoionization edge actually has a multiplet structure owing to the number of $J$ values of both the photoionizing term and of the parent term. In addition, there are series of lines going to high quantum number $n$ that converge at infinity on the parent levels and merge into continua. The line cores overlap and appear to form a continuum before the actual continuum is reached. The location of this point depends on the effective broadening half-widths of the lines, which depend on the physical conditions. It follows that a photoionization edge for a term has the width of the multiplet structure plus the smoothing from the merged lines. It is not a single sharp feature.

Kurucz' programs like SYNTHE (Kurucz \& Avrett 1981; Kurucz 1993b) for computing synthetic spectra, ATLAS9 (Kurucz 1993a) for computing model atmospheres with the Opacity Distribution Functions (ODFs) method, and ATLAS12 (Kurucz 1996) for computing model atmospheres with the opacity sampling method attempt to physically model this structure. For H I, He I, He II, C I, Mg I, Al I, and Si I the line lists are computed or extrapolated to high $n$ for each series of lines that converge on a continuum. The broadening is computed for each line until the line cores overlap. Then a continuum is extrapolated to the continuum edge for that series. For the iron group elements there has not been enough laboratory data to extrapolate or enough computing power to generate such line lists. In addition, autoionizing lines that interact with the continuum can be treated with Fano profiles.

In all the above Kurucz codes b-f cross section data are taken from the literature with none or only the very largest resonance structures. The programs use the cross sections only for computing mass absorption coefficients, not for computing radiative rates. If a cross section has a negligible contribution to the total at a given wavelength, it does not matter what its value actually is. For Fe I, Kurucz made up ad hoc approximate cross sections for the 48 lowest terms that are bell-shaped with a peak of $3 \mathrm{Mb}$ centered $3000 \mathrm{~cm}^{-1}$ into the continuum and that tail off to the red and blue (see Fig. 1, lower panels, 
Table 1. Photoionization data for Fe I. The Fe I ionization potential is $\chi_{\text {ion }}=63737 \mathrm{~cm}^{-1}$.

\begin{tabular}{|c|c|c|c|c|c|c|c|c|c|c|c|c|c|c|}
\hline \multirow[b]{3}{*}{ (1) } & & & \multicolumn{6}{|c|}{ ATLAS9, ATLAS12 (K) } & \multicolumn{2}{|c|}{ This paper (CK) } & \multicolumn{4}{|c|}{ Bautista (B) } \\
\hline & \multicolumn{2}{|c|}{ Fe I config. } & \multirow{2}{*}{$\begin{array}{l}g_{n} \\
(3)\end{array}$} & \multirow{2}{*}{$\begin{array}{c}E_{n} \\
\mathrm{~cm}^{-1} \\
(4)\end{array}$} & \multirow{2}{*}{$\begin{array}{c}\text { Parent } \\
\text { (5) }\end{array}$} & \multirow{2}{*}{$\begin{array}{c}E_{\text {ion }} \\
\mathrm{cm}^{-1} \\
(6)\end{array}$} & \multirow{2}{*}{$\begin{array}{c}\lambda_{\text {ion }} \\
\AA \\
(7)\end{array}$} & \multirow{2}{*}{$\begin{array}{c}E_{n} \\
\mathrm{~cm}^{-1} \\
(8)\end{array}$} & \multirow{2}{*}{$\begin{array}{l}\text { Parent } \\
\text { (9) }\end{array}$} & \multirow{2}{*}{$\begin{array}{c}E_{\text {ion }} \\
\mathrm{cm}^{-1} \\
(10)\end{array}$} & \multirow{2}{*}{$\begin{array}{c}\lambda_{\text {ion }} \\
\AA \\
(11)\end{array}$} & \multirow{2}{*}{$\begin{array}{c}E_{\text {ion }} \\
\mathrm{cm}^{-1} \\
(12)\end{array}$} & \multirow{2}{*}{$\begin{array}{c}\lambda_{\text {ion }} \\
\AA \\
(13)\end{array}$} & $\lambda_{\mathrm{CK}}-\lambda_{\mathrm{B}}$ \\
\hline & (2) & & & & & & & & & & & & & (14) \\
\hline 1 & $3 d^{6} 4 s^{2}$ & $a^{5} D$ & 25 & 500 & $a^{6} D$ & 63500 & 1575 & 403 & $a^{6} D$ & 63334 & 1579 & 62965 & 1588 & -9 \\
\hline 2 & $3 d^{7} 4 s$ & $a^{5} \mathrm{~F}$ & 35 & 7500 & $a^{4} F$ & 58500 & 1709 & 7460 & $a^{6} D$ & 56227 & 1777 & 58523 & 1709 & +68 \\
\hline 3 & $3 d^{7} 4 s$ & $a^{3} \mathrm{~F}$ & 21 & 12500 & $a^{4} F$ & 53500 & 1869 & 12407 & $a^{4} \mathrm{~F}$ & 53327 & 1875 & 54417 & 1838 & +37 \\
\hline 4 & $3 d^{7} 4 s$ & $a^{5} \mathrm{P}$ & 15 & 17500 & $\mathrm{a}^{4} \mathrm{P}$ & 59500 & 1681 & 17685 & $a^{6} D$ & 46052 & 2171 & 47471 & 2107 & +64 \\
\hline 5 & $3 d^{6} 4 s^{2}$ & $\mathrm{a}^{3} \mathrm{P} 2$ & 9 & 19000 & $a^{6} D$ & 45000 & 2222 & 18954 & $a^{4} \mathrm{~F}$ & 46780 & 2138 & 45088 & 2218 & -80 \\
\hline 6 & $3 d^{6} 4 s 4 p$ & $\mathrm{z}^{7} \mathrm{D}^{0}$ & 35 & 19500 & $a^{6} D$ & 44500 & 2247 & 19624 & $a^{6} D$ & 44113 & 2267 & 46098 & 2169 & +98 \\
\hline 7 & $3 d^{6} 4 s^{2}$ & $a^{3} \mathrm{H}$ & 33 & 19500 & $a^{6} D$ & 44500 & 2247 & 19576 & $a^{6} D$ & 44161 & 2264 & & & \\
\hline 8 & $3 d^{6} 4 s^{2}$ & $\mathrm{~b}^{3} \mathrm{~F} 2$ & 21 & 21000 & $a^{6} D$ & 43000 & 2326 & 20814 & $a^{4} \mathrm{~F}$ & 44920 & 2226 & 40788 & 2452 & -226 \\
\hline 9 & $3 d^{7} 4 s$ & $a^{3} G$ & 27 & 22000 & $a^{2} G$ & 58000 & 1724 & 21949 & $\mathrm{a}^{4} \mathrm{~F}$ & 43785 & 2284 & & 2331 & -47 \\
\hline 10 & $3 d^{6} 4 s 4 p$ & $\mathrm{z}^{7} \mathrm{~F}^{0}$ & 49 & 23000 & $a^{6} D$ & 41000 & 2439 & 22928 & $a^{6} D$ & 40809 & 2450 & 42997 & 2326 & +124 \\
\hline 11 & $3 d^{7} 4 s$ & $b^{3} \mathrm{P}$ & 9 & 23000 & $\mathrm{a}^{4} \mathrm{P}$ & 54000 & 1852 & 22898 & $a^{4} \mathrm{~F}$ & 42836 & 2334 & 42001 & 2381 & -47 \\
\hline 12 & $3 d^{6} 4 s 4 p$ & $\mathrm{z}^{7} \mathrm{P}^{0}$ & 21 & 24000 & $a^{6} D$ & 40000 & 2500 & 24057 & $a^{6} D$ & 39680 & 2520 & 41885 & 2387 & +133 \\
\hline 13 & $3 d^{6} 4 s^{2}$ & $b^{3} \mathrm{G}$ & 27 & 24000 & $a^{6} D$ & 40000 & 2500 & 24039 & $a^{4} \mathrm{~F}$ & 41695 & 2398 & 35043 & 2854 & -456 \\
\hline 14 & $3 d^{7} 4 s$ & $c^{3} \mathrm{P}$ & 9 & 24500 & $\mathrm{a}^{2} \mathrm{P}$ & 57500 & 1739 & 24565 & $a^{4} \mathrm{~F}$ & 41169 & 2429 & 40407 & 2475 & -46 \\
\hline 15 & $3 d^{7} 4 s$ & $a^{1} G$ & 9 & 24500 & $a^{2} G$ & 55500 & 1818 & 24575 & $a^{2} G$ & 54822 & 1824 & 48285 & 2071 & -247 \\
\hline 16 & $3 d^{6} 4 s 4 p$ & $z^{5} D^{0}$ & 25 & 26000 & $a^{6} D$ & 38000 & 2632 & 26151 & $a^{6} D$ & 37586 & 2660 & 40222 & 2486 & +174 \\
\hline 17 & $3 d^{7} 4 s$ & $\mathrm{~b}^{3} \mathrm{H}$ & 33 & 26500 & $\mathrm{a}^{2} \mathrm{H}$ & 57500 & 1739 & 26330 & $a^{6} D$ & 37407 & 2673 & & & \\
\hline 18 & $3 d^{7} 4 s$ & $a^{3} D$ & 15 & 26500 & $\mathrm{a}^{2} \mathrm{H}$ & 57500 & 1739 & 26446 & $a^{4} \mathrm{~F}$ & 39288 & 2545 & 61 & 2593 & -48 \\
\hline 19 & $3 d^{6} 4 s 4 p$ & $z^{5} F^{0}$ & 35 & 27000 & $a^{6} D$ & 37000 & 2703 & 27219 & $a^{6} D$ & 36518 & 2738 & 38546 & 2594 & +144 \\
\hline 20 & $3 d^{7} 4 s$ & $\mathrm{a}^{1} \mathrm{P}$ & 3 & 27500 & $\mathrm{a}^{2} \mathrm{P}$ & 54500 & 1835 & 27543 & $a^{2} G$ & 51854 & 1928 & 52656 & 1899 & +29 \\
\hline 21 & $3 d^{7} 4 s$ & $a^{1} D$ & 5 & 28500 & $\mathrm{a}^{2} \mathrm{P}$ & 53500 & 1869 & 28605 & $a^{2} G$ & 50792 & 1969 & 50865 & 1966 & +3 \\
\hline 22 & $3 d^{7} 4 s$ & $\mathrm{a}^{1} \mathrm{H}$ & 11 & 29000 & $\mathrm{a}^{2} \mathrm{H}$ & 55000 & 1818 & 28820 & $a^{2} G$ & 50577 & 1977 & 48310 & 2070 & -93 \\
\hline 23 & $3 d^{6} 4 s 4 p$ & $\mathrm{z}^{5} \mathrm{P}^{0}$ & 15 & 29500 & $a^{6} D$ & 34500 & 2899 & 29329 & $a^{6} D$ & 34408 & 2906 & 36952 & 2706 & +200 \\
\hline 24 & $3 d^{6} 4 s^{2}$ & $\mathrm{a}^{1} \mathrm{I}$ & 13 & 29500 & $a^{6} D$ & 34500 & 2899 & 29313 & $a^{2} G$ & 50084 & 1997 & 47173 & 2120 & -123 \\
\hline 25 & $3 d^{6} 4 s^{2}$ & $b^{3} \mathrm{D}$ & 15 & 29500 & $a^{6} D$ & 34500 & 2899 & 29356 & $a^{6} D$ & 34381 & 2909 & 27290 & 3664 & -755 \\
\hline 26 & $3 d^{6} 4 s^{2}$ & $\mathrm{~b}^{1} \mathrm{G} 2$ & 9 & 30000 & $a^{6} D$ & 34000 & 2941 & 29799 & $a^{2} G$ & 49598 & 2016 & 49032 & 2039 & -23 \\
\hline 27 & $3 d^{6} 4 s 4 p$ & $\mathrm{z}^{3} \mathrm{~F}^{0}$ & 21 & 31500 & $a^{6} D$ & 32500 & 3077 & 31709 & $a^{4} \mathrm{~F}$ & 34025 & 2939 & 35083 & 2850 & +89 \\
\hline 28 & $3 d^{6} 4 s 4 p$ & $\mathrm{z}^{3} \mathrm{D}^{0}$ & 15 & 31500 & $a^{6} D$ & 32500 & 3077 & 31567 & $a^{4} \mathrm{~F}$ & 34167 & 2927 & 36147 & 2766 & +161 \\
\hline 29 & $3 \mathrm{~d}^{8}$ & $c^{3} \mathrm{~F}$ & 21 & 33500 & $a^{6} D$ & 32500 & 3077 & 33266 & $\mathrm{a}^{4} \mathrm{~F}$ & 32468 & 3081 & 25238 & 3962 & -881 \\
\hline 30 & $3 d^{7} 4 p$ & $y^{5} D^{0}$ & 25 & 33500 & $a^{4} \mathrm{~F}$ & 32500 & 3077 & 33504 & $a^{6} D$ & 30233 & 3308 & 31288 & 3196 & +112 \\
\hline 31 & $3 d^{7} 4 p$ & $\mathrm{y}^{5} \mathrm{~F}^{0}$ & 35 & 34000 & $a^{4} \mathrm{~F}$ & 32000 & 3125 & 34118 & $a^{6} D$ & 29619 & 3376 & 30906 & 3235 & +141 \\
\hline 32 & $3 d^{6} 4 s 4 p$ & $\mathrm{z}^{3} \mathrm{P}^{0}$ & 9 & 34500 & $a^{6} D$ & 29500 & 3390 & 34153 & $a^{4} \mathrm{~F}$ & 31581 & 3166 & 33303 & 3003 & +163 \\
\hline 33 & $3 d^{6} 4 s^{2}$ & $\mathrm{~b}^{1} \mathrm{D} 2$ & 5 & 34500 & $a^{6} D$ & 29500 & 3390 & 34637 & $a^{2} G$ & 44760 & 2234 & 43982 & 2274 & -40 \\
\hline 34 & $3 d^{7} 4 p$ & $z^{5} G^{0}$ & 45 & 35000 & $a^{4} F$ & 31000 & 3226 & 35143 & $a^{6} D$ & 28594 & 3497 & 29671 & 3370 & +127 \\
\hline 35 & $3 d^{7} 4 p$ & $\mathrm{z}^{3} \mathrm{G}^{0}$ & 27 & 35500 & $a^{4} F$ & 30500 & 3279 & 35690 & $a^{4} \mathrm{~F}$ & 30044 & 3328 & 30881 & 3238 & +90 \\
\hline 36 & $3 d^{7} 4 p$ & $\mathrm{y}^{3} \mathrm{~F}^{0}$ & 21 & 37000 & $a^{4} \mathrm{~F}$ & 29000 & 3448 & 37043 & $a^{4} \mathrm{~F}$ & 28691 & 3485 & 29145 & 3431 & +54 \\
\hline 37 & $3 d^{6} 4 s 4 p$ & $\mathrm{y}^{5} \mathrm{P}^{0}$ & 15 & 37000 & $a^{6} D$ & 27000 & 3704 & 37026 & $a^{6} D$ & 26711 & 3744 & 26977 & 3707 & +37 \\
\hline 38 & $3 d^{7} 4 s$ & $\mathrm{~d}^{3} \mathrm{~F}$ & 21 & 37000 & $b^{2} \mathrm{~F}$ & 54000 & 1852 & 36997 & $a^{4} \mathrm{~F}$ & 28737 & 3480 & 17656 & 5664 & -2184 \\
\hline 39 & $3 d^{7} 4 p$ & $\mathrm{y}^{3} \mathrm{D}^{0}$ & 15 & 38500 & $a^{4} \mathrm{~F}$ & 27500 & 3636 & 38507 & $a^{4} F$ & 27227 & 3673 & 27368 & 3654 & +19 \\
\hline 40 & $3 d^{6} 4 s 4 p$ & $x^{5} D^{0}$ & 25 & 40000 & $a^{6} D$ & 24000 & 4167 & 39971 & $a^{6} D$ & 23766 & 4208 & 24541 & 4075 & +133 \\
\hline 41 & $3 d^{5} 4 s^{2} 4 p$ & $\mathrm{y}^{7} \mathrm{P}^{0}$ & 21 & 40000 & $a^{6} S$ & 47000 & 2128 & 40262 & $a^{6} D$ & 23475 & 4260 & 24346 & 4107 & +153 \\
\hline 42 & $3 d^{6} 4 s 4 p$ & $\mathrm{x}^{5} \mathrm{~F}^{0}$ & 35 & 41000 & $a^{6} D$ & 23000 & 4348 & 40645 & $a^{6} D$ & 23092 & 4330 & 24450 & 4090 & +240 \\
\hline 43 & $3 d^{6} 4 s 4 p$ & $z^{5} S^{0}$ & 5 & 41000 & $b^{4} \mathrm{P}$ & 44000 & 2273 & 40895 & $a^{6} D$ & 22842 & 4378 & 22447 & 4455 & -77 \\
\hline 44 & $3 d^{6} 4 s 4 p$ & $\mathrm{x}^{5} \mathrm{P}^{0}$ & 15 & 43000 & $b^{4} \mathrm{P}$ & 42000 & 2273 & 42751 & $a^{6} D$ & 20986 & 4765 & 19894 & 5027 & -262 \\
\hline 45 & $3 d^{6} 4 s 4 p$ & $y^{5} G^{0}$ & 45 & 43000 & $b^{4} \mathrm{P}$ & 42000 & 2273 & 42965 & $a^{6} D$ & 20772 & 4814 & 17839 & 5606 & -792 \\
\hline 46 & $3 d^{6} 4 s 5 s$ & $\mathrm{e}^{7} \mathrm{D}$ & 35 & 43000 & $a^{6} D$ & 21000 & 4762 & 43227 & $a^{6} D$ & 20510 & 4876 & 20717 & 4827 & +49 \\
\hline 47 & $3 d^{6} 4 s 4 p$ & $\mathrm{z}^{5} \mathrm{H}^{0}$ & 55 & 43000 & $b^{4} \mathrm{P}$ & 42000 & 2273 & 43184 & $a^{6} D$ & 20553 & 4865 & 17064 & 5860 & -995 \\
\hline 48 & $3 d^{6} 4 s 4 p$ & $w^{5} D^{0}$ & 25 & 44000 & $b^{4} \mathrm{P}$ & 42000 & 2273 & 43903 & $a^{6} D$ & 19834 & 5042 & 19441 & 5144 & -102 \\
\hline
\end{tabular}

yellow line). The multiplet structure is smoothed away. The red tail accounts for the missing high- $n$ lines. The blue tail drops off faster than the real cross section, but it does not matter to the total opacity because there are so many levels. The $3 \mathrm{Mb}$ estimate was made from looking at the computed cross sections of Dragon \& Mutschlecner (1980) for the low Fe I terms. They fitted calculations by Merts \& Matuska (1972). Kurucz mistakenly thought that $3 \mathrm{Mb}$ was a good estimate of the peak cross 

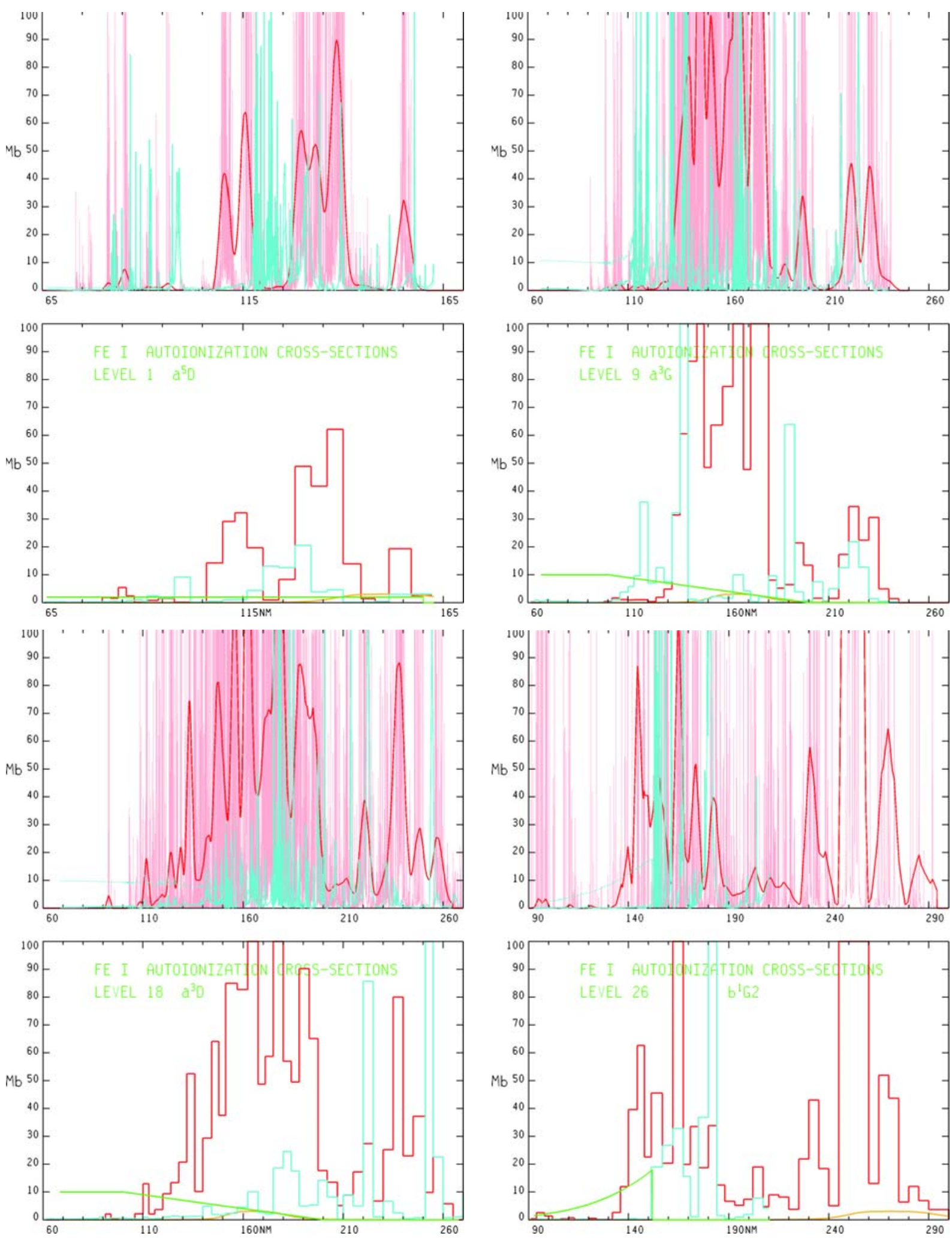

Fig. 1. Comparison of cross sections $\sigma_{n}(v)$ as function of $\lambda$ for the Fe I terms indicated in Table 1 with numbers 1, 9, 18, 26 (in this page), 34, and 42 (in the next page). For each term there are two panels. In the upper panel Bautista (thick blue) and Kurucz (pink) cross sections of autoionization lines are compared. The whole Bautista cross section (thin blue) and the smoothed cross section of autoionization lines from Kurucz (red) are also plotted. In the lower panel the Bautista estimated continuum (green) is compared with the continuum from Kurucz (yellow). Average line cross sections of the autoionization lines from Bautista (blue) for each "big" wavelength interval in the ODFs are compared with those from Kurucz (red). 

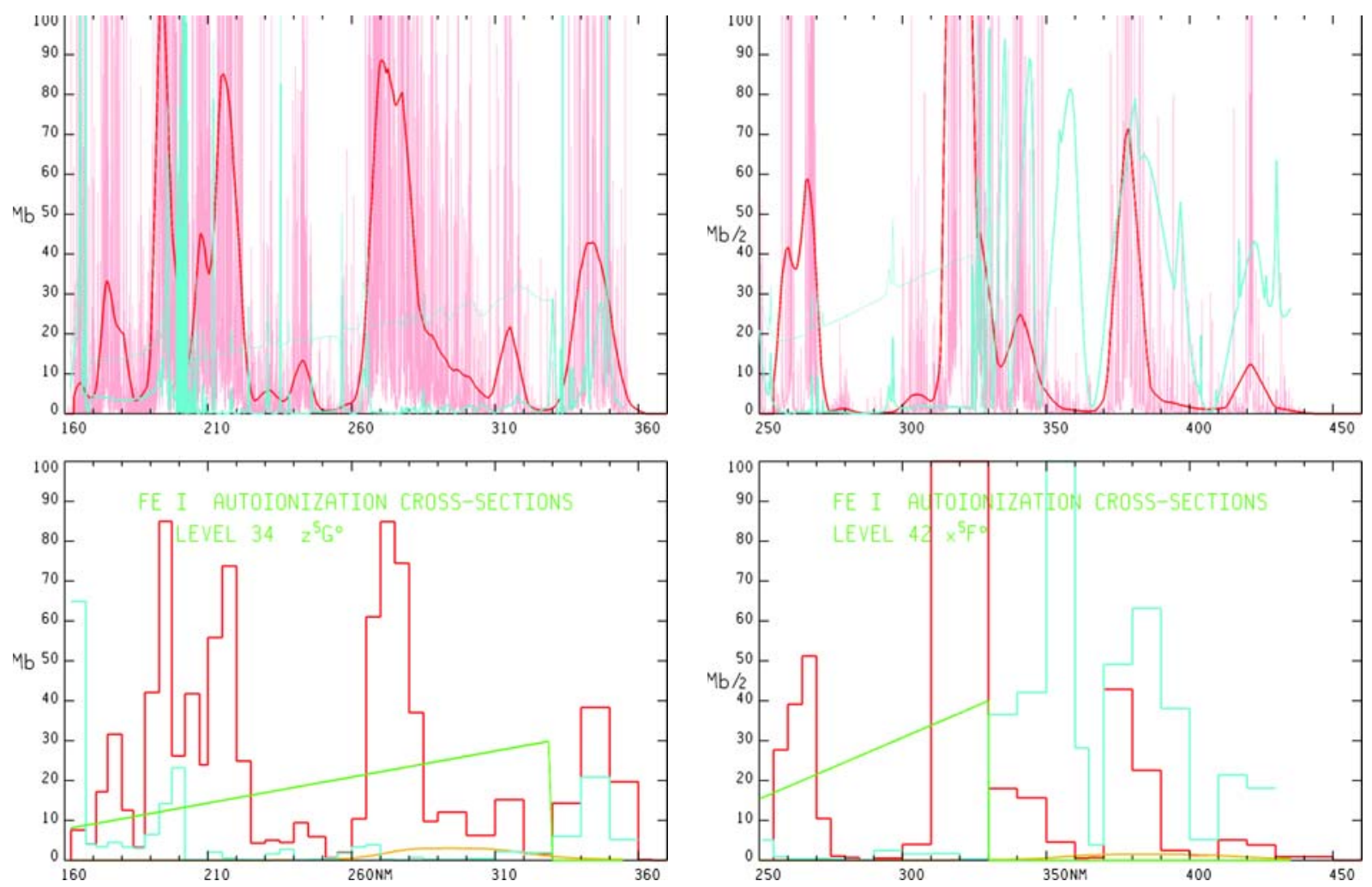

Fig. 1. continued.

section for each term. In fact, in their paper the largest cross section is $6.6 \mathrm{Mb}$ at the $3750 \AA$ threshold for the $\mathrm{y}^{5} \mathrm{P}$ term, but that edge is not visible in the sun, so Kurucz thought it did not matter.

\subsection{Fe I photoionization cross sections from Bautista}

The Fe I cross sections computed by Bautista (1997) are sampled with a frequency resolution approximately 1000 points per rydberg and they include both b-f transitions and autoionizing resonances (Fig. 1, upper panels, thin blue line). The irregular shape of the cross sections and the crowding of autoionizing resonances hamper any use of polynomial functions approximating the cross section behaviour. To reduce the huge number of the cross section points which would enter into ATLAS9 computations, we averaged the Bautista cross sections over the Opacity Distribution Function (ODF) "big" wavelength intervals.

\subsection{Fe I autoionizing lines in LOWLINES}

When the cross sections from Bautista (1997) (Fig. 1, upper panels, thin blue colour) are compared with the b-f cross sections from Kurucz (Fig. 1, lower panels, yellow colour) the difference is striking, but we want to remark that while autoionization lines are included in the Bautista cross sections, they are not part of the ATLAS9 b-f opacity. However, because a large number of Fe I lines having the upper level higher than the first ionization limit contribute to the total opacity of models and fluxes, we have added their cross sections to the b-f cross sections from Kurucz and have compared the corresponding absorption coefficients with absorption coefficients computed with the Bautista cross sections. These lines are part of the Kurucz (1994) LOWLINES line list. We will indicate them as autoionizing lines, although interactions of their discrete upper level with the continuum were not considered in the Kurucz computations.

To compute the iron group line lists Kurucz (1988) made Slater-expansion model Hamiltonians that included as many configurations as he could fit into the Cray. He used HartreeFock Slater integrals (scaled) for starting guesses and higher configurations that had no laboratory energy levels. All (boundstate) configuration interactions were included. He then determined the Slater integrals for the observed configurations by least squares fitting the eigenvalues computed for the Hamiltonian matrix to the observed energies. The complication was that the eigenvalues and the observed energies had to be correlated by hand each iteration and more than a hundred iterations were often required for convergence. The computer programs for these procedures evolved from Cowan's (1968) programs. Transition integrals were computed with scaled-Thomas-Fermi-Dirac wavefunctions and the whole transition array was produced for each ion. Radiative, Stark, and van der Waals damping constants and Landé $g$ values were automatically produced for each line. Eigenvalues were replaced by measured energies so that lines connecting measured levels have correct wavelengths. Most of the lines have uncertain wavelengths because they connect predicted rather than measured levels.

Rigorously, the cross sections for autoionizing lines and the continua must be computed together, but, in practice, the lines and continua can be treated independently when interactions 
do not produce deep broad minima in the continuum and large widths and asymmetries in the absorption profiles. Figure 1 (upper panels, thin blue line) indicates that the Bautista cross sections do not show remarkable continuum depressions and that only small asymmetry effects compared to the much larger line opacity are present (Fig. 1, upper panels, thick blue line). Inspection of Bautista's cross sections for the other terms listed in Table 1 has shown a similar behaviour.

If the asymmetry does not dominate the continuum, it can be ignored because the positive and negative contributions to the profile approximately cancel, so that the $g f$ value is independent of the profile. An arbitrary Voigt or Lorentzian profile can be used to compute the spectrum of the line cross section when the cross section is used statistically. Here, we made this assumption for the Fe I autoionization lines. We note that the Kurucz programs allow widths and asymmetries to be assigned to each autoionizing line so that it can be computed with a Fano line profile. For a few elements, some autoionizing lines in Kurucz's line list have measured parameters and are treated in this way.

The autoionization cross section for each term was produced by computing the mass absorption coefficient spectrum for one temperature-pressure layer of a solar model using program SYNTHE. Only Fe I lines in Kurucz's linelist arising from that term with an upper level greater than the ionization limit $63737 \mathrm{~cm}^{-1}$ were used as input. The program was run as for a normal spectrum calculation at resolution 500000 but with the radiative damping $\gamma_{\text {rad }}$ set to $10^{12}$ for the Voigt profile and with the wing cutoff reduced by 100 to make the wings more realistic. Then the mass absorption coefficient spectrum was divided by the number of iron atoms populating that term per gram to get the average cross section spectrum for that term.

Bautista (1997) has computed photoionization cross sections for Fe I for continua and autoionizing lines together. To make the problem tractable he assumed that each term has only one level with its average energy and he computed the total cross section spectrum for each term. He did not tabulate the positions, widths, and asymmetries of the autoionization lines. Here we estimate the continua from the tabulated cross section spectra by removing the asymmetries with a marking pen. The dips are only a small fraction of the total opacity and the "smooth continuum" can be easily fitted by simple functions (i.e. Dragon \& Mutschlecner 1980). Then, we subtract the continuum out leaving the autoionizing line spectrum. We can then separately compare Kurucz and Bautista continuum (Fig. 1, lower plot, yellow and green colours, respectively) and autoionizing line contributions to the total opacity (Fig. 1, pink and red colours show Kurucz's lines, thick blue colour shows Bautista's lines).

The most impressive differences are the much larger number of lines in Kurucz than in Bautista and the shifts of the red and blue peaks relative to each other. We can explain the first difference with the fact that Kurucz treats every $J$ level of Fe I including most of the levels up through $n=9$, i.e. about 39000 levels, and considers all the lines they produce, while autoionization lines in Bautista are term-transitions. The second difference can be due to systematic errors in the heights of predicted configurations in Kurucz, in Bautista, or in both.

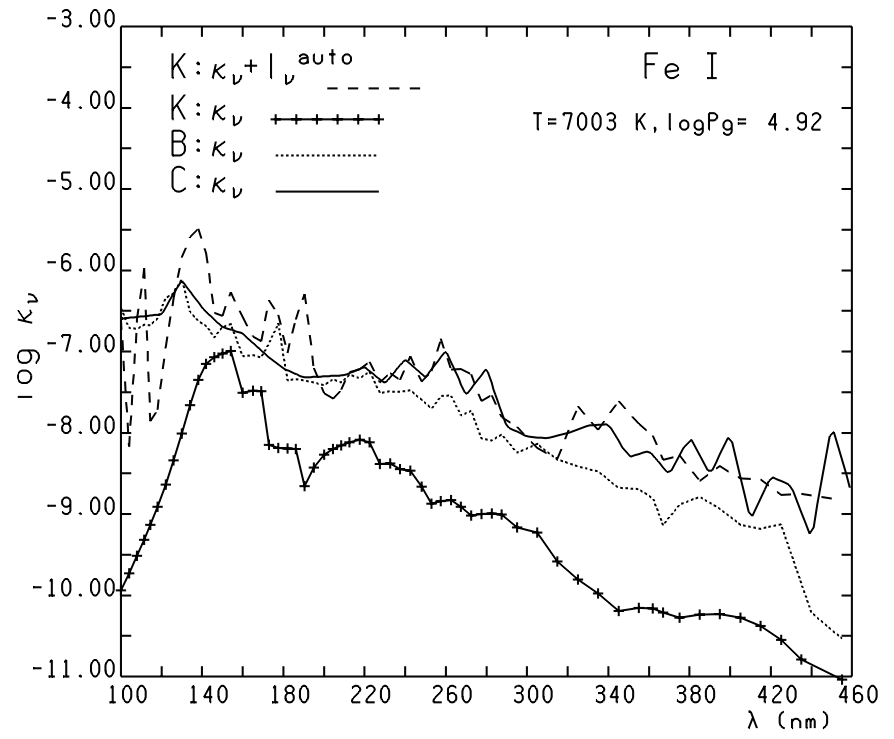

Fig. 2. Comparison of Fe I opacities: (1) $\mathrm{K}: \kappa_{v}+1_{v}^{\text {auto }}$ is the sum of the Fe I b-f ATLAS9 opacity with the line opacity due to the only Fe I autoionization lines from LOWLINES (Kurucz 1994) (dashed line); (2) $\mathrm{K}: \kappa_{v}$ is the b-f Fe I opacity from ATLAS9 (line with plus); (3) B: $\kappa_{v}$ is the opacity computed with the Bautista (1997) cross sections (dotted line); (4) C: $\kappa_{v}$ is the opacity computed by Cowley with the K-factor method (Cowley \& Bautista 2003) (full line). Absorption coefficients are in $\mathrm{cm}^{-1}$.

Kurucz used all the laboratory energy levels that existed in a semiempirical calculation and predicted the rest. Some wavelengths and photoionization edges should have correct wavelengths. Kurucz hopes to improve the predicted levels in new calculations. The Bautista calculations are self-consistent so they do not reproduce observed level positions. For the lower terms, the Bautista and Kurucz photoionization continua are of similar scale where they matter. For the higher terms Bautista is an order of magnitude larger. It would be straightforward to replace all the Kurucz cross sections with simple fits to Bautista like those in Fig. 1.

\section{Comparison of absorption coefficients}

In order to quantify the effect of the Fe I autoionizing lines on the total Fe I opacity, we extracted from the LOWLINES line list (Kurucz 1994) all Fe I lines having the energy of the upper level larger than the Fe I ionization energy; then, for a given combination $T, \log P_{\text {gas }}$ we computed the line opacity spectrum in the wavelength interval 800-5000 $\AA$ at 500000 resolution. The line opacity was then averaged over the ODF "big" wavelength intervals. Finally, the average line opacity of the $\mathrm{Fe}$ I autoionization lines $\mathrm{l}_{v}^{\text {auto }}$ was added to the continuous Fe I opacity $\kappa_{v}$.

Figure 2 compares the summed opacity (dashed line) with the absorption coefficient computed with the Bautista cross sections (dotted line) and with the b-f coefficient from ATLAS9 (line with plus). While $\kappa_{v}$ from Bautista is more than ten times larger than $\kappa_{v}$ from ATLAS9, the difference between $\kappa_{v}$ from Bautista and the summed opacity is considerably reduced, thus indicating the importance of the Fe I line opacity due to lines 


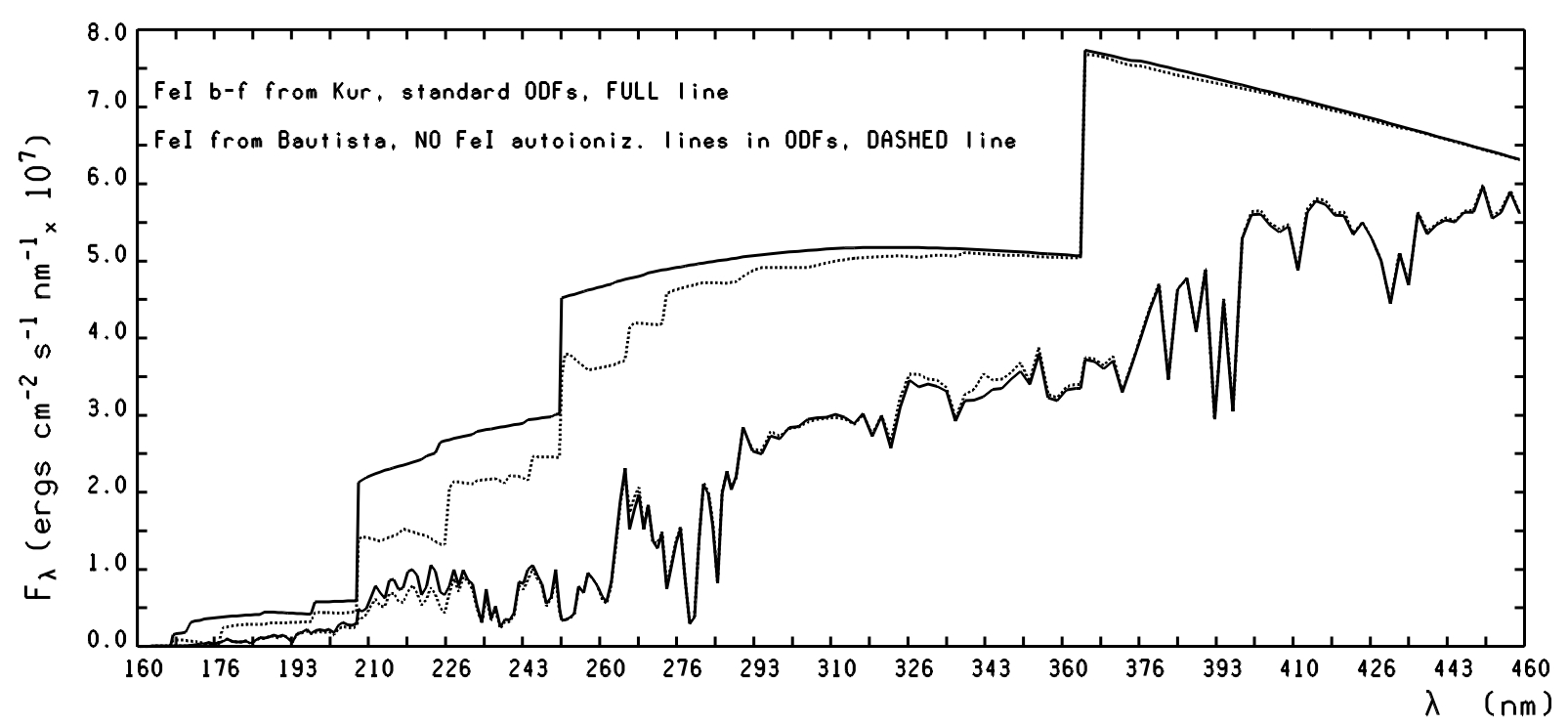

Fig. 3. A standard ATLAS9 flux computed from a standard ATLAS9 model (full lines) is compared with a flux computed with Bautista Fe I cross sections and no Fe I autoionization lines in the ODFs (dashed lines). Bautista has more opacity from 208 to 228 nm while Kurucz has more opacity from 326 to $350 \mathrm{~nm}$, but generally they are similar. Model parameters are $T_{\text {eff }}=6500 \mathrm{~K}, \log g=4.5,[\mathrm{M} / \mathrm{H}]=0.0, \xi=2 \mathrm{~km} \mathrm{~s}{ }^{-1}$. The two upper lines compare the continua, the two lower lines compare the emergent fluxes.

with the upper level above the ionization threshold. The behaviour shown in Fig. 2 for $T=7003 \mathrm{~K}, \log P_{\text {gas }}=4.92 \mathrm{did}$ not change in a significant way for other $\left(T, \log P_{\text {gas }}\right)$ couples examined.

We have also compared the summed opacity with the Fe I absorption coefficient computed by Cowley (2002, private communication) by means of the K-factor method and all the Bautista cross sections, which were computed for a very large number of terms (Cowley \& Bautista 2002). Following Peach (1967), this method consists in computing a weighted sum of cross sections sampled at small wavelength intervals (for instance $20 \AA$ ) over all the terms with available cross sections. The opacity for the wavelengths and temperatures entering into model or flux computations is obtained by a linear interpolation in the temperature-wavelength grids. Figure 2 shows the close agreement between the summed opacity computed by us (dashed line) and the Fe I opacity computed by Cowley (full line).

\section{Influence of Fe I opacity on model atmospheres and fluxes}

In order to assess the effect of different Fe I opacities on model atmospheres and fluxes we substituted in ATLAS9 the Fe I continuous opacity from Kurucz with the Fe I opacity computed with the Bautista cross sections averaged over the ODF "big" wavelength intervals; then, we dropped all the Fe I lines with the upper level above the Fe I ionization energy in the LOWLINES line list (Kurucz 1994) and we computed solar abundance ODFs with this new line list.

The new Fe I continuous opacity and the new ODFs were used to generate a few model atmospheres and energy distributions for $T_{\text {eff }}$ from $4000 \mathrm{~K}$ to $9000 \mathrm{~K}$ and several different $\log g$. The comparison of the $T-\tau_{\text {Ross }}$ relation from these models with the corresponding relations from standard ATLAS9 models did not show any difference, while the comparison of fluxes pointed out small differences in the regions 2000-2300 $\AA$ and 3250-3450 $\AA$, but limited to models with $T_{\text {eff }} \leq 7500 \mathrm{~K}$. The largest differences occur for $T_{\text {eff }}$ around $6000-6500 \mathrm{~K}$. Figure 3 compares fluxes with parameters $T_{\text {eff }}=6500 \mathrm{~K}, \log g=4.5,[\mathrm{M} / \mathrm{H}]=0.0$ and microturbulent velocity $\xi=2 \mathrm{~km} \mathrm{~s}^{-1}$. The figure shows both the continuous flux and the emergent flux. We note that at $3300 \AA$ the standard ATLAS9 emergent flux is lower than that based on the Bautista cross sections, while the converse is true at $2150 \AA$. The differences are of the order of $23 \%$ at $2150 \AA$ and of $8 \%$ at $3350 \AA$. Because there is a fall of the flux at 2000-2300 in cool stars, every difference disappears in this wavelength region for $T_{\text {eff }} \leq 5500 \mathrm{~K}$. A change of the gravity does not modify these results.

The effect of the Fe I autoionization lines can be seen in Fig. 4, where fluxes which differ only for the ODFs adopted in the computations are compared. In one case ODFs include the Fe I autoionization lines, they do not in the other case. While, as expected, the continuous flux is the same for the two models, the contribution of the Fe I autoionization lines reduces the emergent flux mostly shortward of the Balmer discontinuity and especially around $3300 \AA, 2700 \AA$, and $2200 \AA$, where the flux difference is of the order of $6 \%, 13 \%$, and $20 \%$, respectively. The increase of the percentage difference with decreasing wavelength is proportional to the fall of the emitted radiation.

The effect of Fe I continuous opacity can be inferred from Fig. 5 where we compared the flux computed with Fe I continuous cross sections from Kurucz (Sect. 3.1) with that computed with the Bautista cross sections (Sect. 3.2). In both models the line opacity is contributed by ODFs not including Fe I autoionization lines. The figure shows that when the Fe I Bautista 


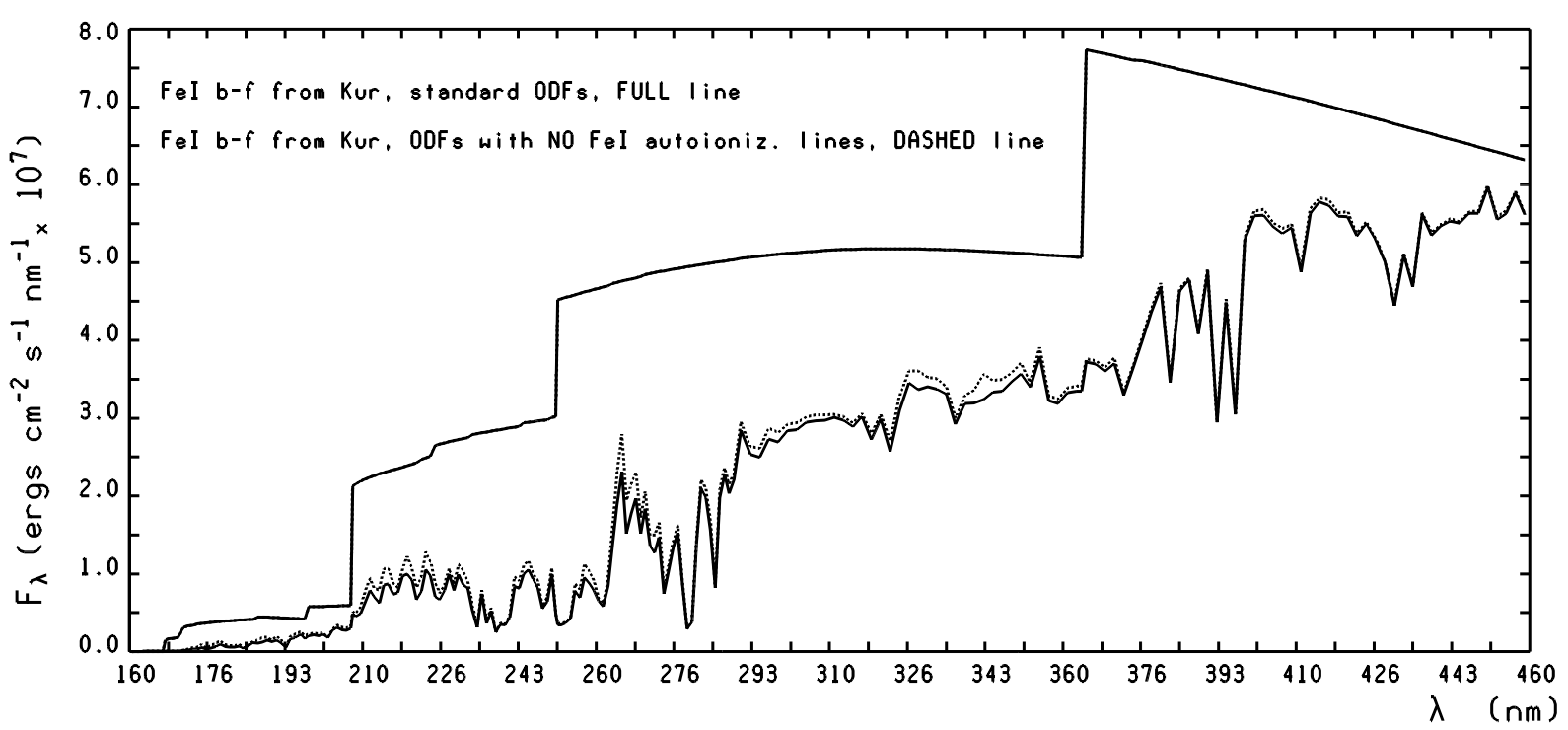

Fig. 4. Comparison of fluxes computed with and without Fe I autoionization lines which produce a few percent of the total opacity in this wavelength interval. The two fluxes were computed with different ODF's, but with the same ATLAS9 continuous opacity. Model parameters are $T_{\text {eff }}=6500 \mathrm{~K}, \log g=4.5,[\mathrm{M} / \mathrm{H}]=0.0$, and $\xi=2 \mathrm{~km} \mathrm{~s}^{-1}$. The upper full line is the continuous flux, which is the same in the two cases. The lower full line represents the flux computed with ODFs including Fe I autoionization lines, the dashed line indicates the flux computed with ODFs not including Fe I autoionization lines.

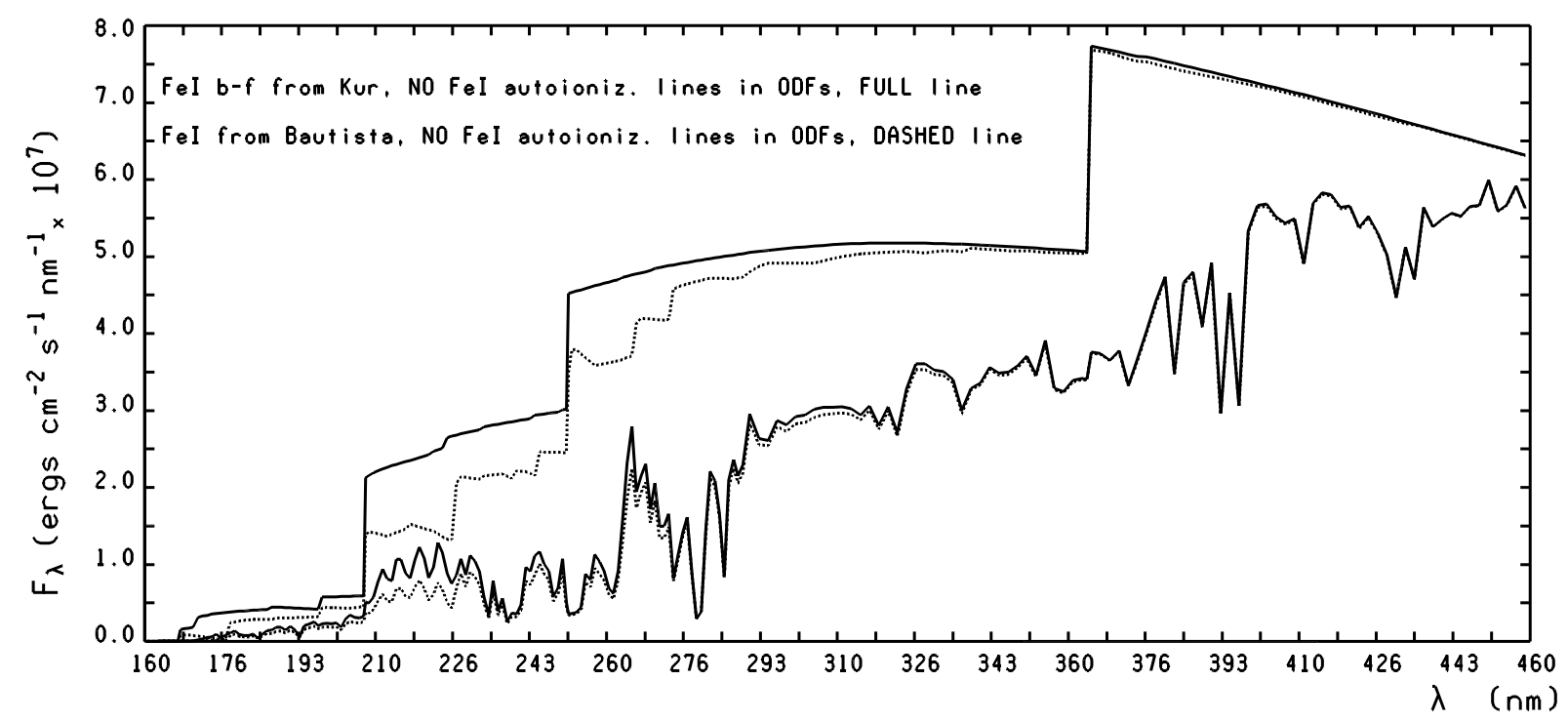

Fig. 5. The flux computed using Fe I b-f cross sections from Kurucz (Sect. 3.1) (full lines) is compared with the flux computed using the averaged Bautista (1997) cross sections (Sect. 3.2) (dashed lines). For both fluxes, ODFs with no Fe I autoionization lines were used. Model parameters are $T_{\text {eff }}=6500 \mathrm{~K}, \log g=4.5,[\mathrm{M} / \mathrm{H}]=0.0$, and $\xi=2 \mathrm{~km} \mathrm{~s}^{-1}$. The two upper lines compare the continua, the two lower lines compare the emergent fluxes.

cross sections are used, the increased continuous opacity lowers not only the continuous flux, but also the emergent flux, although to a lesser extent. The largest difference between the fluxes computed with different Fe I opacities occurs in the region 2100-2300 $\AA$, where strong autoionization lines of the Bautista cross sections lie.

\section{Summary and conclusions}

We have investigated the magnitude of the errors in models and fluxes when Fe I cross sections approximating
Dragon \& Multschlecner (1980) rather than Fe I cross sections from Bautista (1997) are used. We found that the Fe I absorption coefficient is underestimated by more than ten times in the first case. We also showed that the increased Fe I continuous opacity from Bautista (1997) is compensated in the standard ATLAS9 models by the line opacity due to the Fe I autoionization lines.

As a consequence, there are no differences in the $T-\tau_{\text {Ross }}$ relations from standard ATLAS9 models and models computed with Bautista (1997) Fe I cross sections, while flux differences can be seen in the intervals 2000-2300 $\AA$ and 3250-3450 , 
limitedly to models with $T_{\text {eff }}<7000 \mathrm{~K}$. The most significant differences occur for $T_{\text {eff }}$ around $6000-6500 \mathrm{~K}$. For a $[6500,4.5]$ model, the standard ATLAS9 flux at 3250-3450 is $8 \%$ lower than the Bautista (1997) cross sections flux, so indicating the larger total opacity at these wavelengths from standard models. The standard ATLAS9 flux is $23 \%$ higher at 2000-2300 $\AA$, but any difference disappears in this region for $T_{\text {eff }} \leq 5500 \mathrm{~K}$, owing to the fall of emergent flux in cool stars at these wavelengths.

The similar results obtained from standard ATLAS9 models and models computed with the Fe I Bautista (1997) cross sections do not mean that the Fe I opacity does not need further improvements. In fact, comparisons with the observations (Bell et al. 2001); Cowley \& Castelli 2002) indicate that both computations appear to understimate the real Fe I absorption. We expect the opacity of other Fe group elements to be underestimated as well. Errors in the Fe I cross sections should matter in a statistical equilibrium calculation for $\mathrm{Fe}$ I and Fe II.

As far as Kurucz's computations are concerned, they do not consider interactions of lines with continuum so that no depressions in the Fe I continua are predicted and no Fe I autoionization profile is a Fano profile. Furthermore, the computed energy of some levels lying above the ionization threshold have errors due to the least-squares fitting method adopted (Sect. 3.3). It follows that uncertainties are present in both wavelength and $\log g f$ for some transitions. They can be quantified when high-resolution observed spectra are compared with synthetic spectra. The computed autoionization lines may either be shifted in wavelength relative to the observed lines, or may have too strong intensities, or both, so that they may not correspond to the observable counterparts. For all these reasons, the predicted Fe I autoionization lines are not intended to be used for the analysis of high resolution spectra. Their aim is only that of increasing the total opacity involved in the model atmospheres and in the energy distribution computations. The comparison with the models computed with the cross sections from Bautista (1997) has shown that this aim has been achieved.

We conclude that results from ATLAS9 models are almost independent of the choice of the Fe I cross sections adopted in the Kurucz codes or computed by Bautista (1997), provided that in the first case Fe I autoionization lines are kept in the model and flux computations.
Acknowledgements. We thank Manuel Bautista for the whole set of Fe I cross sections that he has made available to us and for his help in using the files. Fiorella Castelli thanks Charles R. Cowley for the very numerous constructive mails and discussions concerning this paper.

\section{References}

Bautista, M. A. 1997, A\&AS, 122, 167

Bautista, M. A., \& Pradhan, A. K. 1995, J. Phys.B: At. Mol. Phys., 28, L173

Bell, R. A., Balachandran, S. C., \& Bautista, M. 2001, ApJ, 546, L65

Corliss, C., \& Sugar, J. 1982, J. Phys. Chem. Ref. Data, 11, 135

Cowan, R. D. 1968, Journ. Opt. Soc. Amer., 58, 808

Cowley, C. R., \& Bautista, M. 2003, MNRAS, 341, 1226

Cowley, C. R., \& Castelli, F. 2002, A\&A, 387, 595

Dragon, J. N., \& Mutschlecner, J. P. 1980, ApJ, 239, 1045

Gustafsson, B., Bell, R. A., Eriksson, K., \& Nordlund, A. 1975, A\&A, 42,407

Houdashelt, M. L., Bell, R. A., \& Sweigart, A. V. 2000, ApJ, 119, 1448

Hummer, D. G., Berrington, K. A., Eissner, W., et al. 1993, A\&A, 279, 298

Kurucz, R. L. 1988, Transactions of the International Astronomical Union, ed. M. McNally, XXB, 168

Kurucz, R. L. 1993a, ATLAS9 Stellar Atmosphere Programs and $2 \mathrm{~km} / \mathrm{s}$ grid, CD-ROM No 13

Kurucz, R. L. 1993b, SYNTHE Spectrum Synthesis Programs and Line Data, CD-ROM No 18

Kurucz, R. L. 1994, Atomic Data for Opacity Calculations, CD-ROM No 1 (revised)

Kurucz, R. L. 1996, in M.A.S.S. Model Atmospheres and Spectrum Synthesis, ed. S. Adelman, F. Kupka, \& W. W. Weiss, ASP Conf. Ser., 108, 160

Kurucz, R. L., \& Avrett, E. H. 1981, SAO Special Report, 391

Merts, A., \& Matuska, W. 1972, Polynomial Solutions of the Schrödinger Equation Applied to Photon Cross Sections in Atoms, Los Alamos Sci. Lab. Report, LA523

Nave, G., Johansson, S., Learner, R. C. M., Thorne, A. P., \& Brault, J. W. 1994, ApJS, 94, 221

Peach, G. 1967, Mem. R. Astron. Soc., 71, 29

Seaton, M. J., Yu, Yan, Mihalas, D., \& Pradhan, A. K. 1994, MNRAS, 266,805 